\title{
The Origin of Ovarian Cancer - Still Debatable
}

\section{Pushpam Kumar Sinha*}

Independent Researcher, Kankarbagh, Patna, India

*Corresponding Author: Pushpam Kumar Sinha, Independent Researcher, Kankarbagh, Patna, India.
Received: August 26, 2021

Published: September 20, 2021

(C) All rights are reserved by Pushpam Kumar Sinha.

\begin{abstract}
Despite the discovery of two types of Adult Stem Cells (ASCs) in ovary, the Very Small Embryonic like Stem Cell and the Ovarian Stem Cell, the debate over the origin of Ovarian Cancer (OC) is far from over. Like several other cancers, Cancer Stem Cells (CSCs) in recent years have been held responsible for tumor initiation and progress in OC too. CSCs are known to originate from malignant transformation (by genetic and or epigenetic mutations) of ASCs and or their multi-potent progenitors. In liver there exists cellular plasticity between ASC and mature differentiated cells (MDCs). Hence, in liver, even MDCs can be the origin of CSCs. I hypothesize in this article that, like liver, even the MDCs of ovary can be the origin of Ovarian CSC. MDCs of ovary transform to CSC by replacing the nuclei of oocytes by their own nuclei.
\end{abstract}

Keywords: Cancer Stem Cells; Very Small Embryonic-like Stem Cells; Ovarian Stem Cells; Cellular Plasticity; Serous Epithelial Ovarian Cancer

\section{Introduction}

Many of the healthy organs in human body harbor within themselves a rare and quiet population of cells having the selfrenewing capability which is essential for the day-to-day normal maintenance of the organ, and replenishment of the organ in times of damage/injury. This population of cells are called Adult Stem Cells (ASCs). The ASCs in some of the organs are not quiet, like, for example that in the intestinal epithelium as they have to continuously replace the old cells of the intestinal lining with the new one. The research conducted over the last 10-15 years has shown that when these types of organs (they who harbor ASCs) turn cancerous, the tumor cells harbor within themselves Cancer Stem Cells (CSCs) which are the sole agents responsible for tumor initiation and progress [1-3]. The CSCs share many common features with
ASCs, and hence they have been isolated, identified and characterized for many cancers including Ovarian Cancer(OC) [4-7].

The main foci of this perspective article are the papers $[4,5]$. The paper [5] establishes beyond doubt that the human ovary harbors ASCs; in fact there are two types of ASCs in human ovary- 2-6 $\mu \mathrm{m}$, very small embryonic-like stem cells (VSELs) and 6-8 4 m ovarian stem cells (OSCs). Over the years there have been numerous other works claiming the existence of pluripotent putative stem cells in mammalian and or adult human ovary [8-11].

For many years it was believed that the human ovary does not harbor ASCs and hence has a fixed reserve of oocyte-containing follicle in the cortex. A research paper published so latest as that in 2020 [12] claimed that the human ovary does not house stem 
cells. The anomaly between this work and that of the other works has been explained in [5] - the researchers in [12] spun the single cell suspension of ovarian tissue obtained after enzymatic digestion at $300 \mathrm{~g}$ where as the spinning speed was supposed to be kept at $1000 \mathrm{~g}$. CSCs in various types of OCs have been identified with markers CD24, CD34, CD44, CD105, CD117, EpCam, ROR1, ALDH, SOX2, OCT4, etc. [4]. The authors in the paper [4] believe in the hypothesis that it is the ASCs of ovary that transform to CSCs by a sequence of non-consistent genetic mutations. But, given the large scale heterogeneities of OC (there is heterogeneity even amongst the patients suffering from histologically similar type of OC), I disagree with them, and propose my own hypotheses of the origin of ovarian CSC.

\section{The hypotheses}

What types of cells are present in the ovary? Apart from the VSELs and OSCs present in human ovary, the Ovarian Surface Epithelium (OSE) houses squamous-to-cuboidal epithelial cells, the cortex houses oocytes, granulosa cells, immune cells, endothelial cells, perivascular cells, and stromal cells [12], and the medulla houses granulosa cells, theca cells and stroma, smooth muscle cells, endothelial cells, and immune cells [13]. My hypothesis on the origin of CSC in OC is that each of these cell types can transform to CSC by a sequence of multi-stage genetic mutations. The malignant transformation of mature differentiated cells (MDC) in the organ (to CSC), like that in liver [14], has been attributed to the ability of MDC to initialize upon regeneration (cellular plasticity). Such plasticity has not been observed in ovary, even though the Epithelial-toMesenchymal transition plasticity has been observed in ovary [15]. So how come my hypothesis holds ? In one study the stem cells of the ovary were successfully differentiated into the cells of all the three primary germ layers, the mesoderm, the ectoderm, and the endoderm [8]. Hence, we can say that all the cells of the ovary stated above, can arise from VSEL and OSC. The somatic cells of the human body can be re-programmed into Pluripotent Stem Cell by replacing the nucleus of the oocyte by that of the somatic cell concerned $[16,17]$. This technique, known as Somatic Cell Nuclear Transfer (SCNT) has been used over several decades to clone animals like sheep, monkey, etc. So here goes my second hypothesis of this paper- after undergoing several stages of genetic mutations the nucleus of the MDC of ovary replace the nucleus of oocyte, and thereby transform to CSC. Though nuclear transfer has not been known till now as a natural process, but my hypothesis claims it to be a natural process (within the ovary) possibly triggered by external factors like unhealthy diet, smoking etc. Ovary is such a small organ (and the precise location of stem cells within ovary is yet unknown) that upon trigger to spontaneously mutate, it is not simply the stem cells that mutate but even the MDC. And because the stem cells populations have been found in OC that initiate, grow, and metastasize tumor; it is highly likely that the CSC has origin in MDC too apart from having it in ASC. Biology is highly complex, anything is possible, but to take research forward one must think out of the box.

\section{The debate}

Having hypothesized the two hypotheses here, the debate over the origin of OC is far from over. The several types of OC are epithelial (serous, endometrioid, clear cell, and mucinous), germ cell, and stromal cells [4]. Out of these the serous epithelial ovarian cancer (SEOC) is the most lethal. Let us discuss the origin of SEOC to understand the complexities involved. For long the origin of SEOC has been known to be Fallopian Tube (FT) [18]. ButCSCs were found also in the ovary in SEOC [18]. So one can debate that the SEOC originated in FT and metastasized along with CSC to ovary, or it originated in ovary and metastasized to FT. The discovery of CSC within the ovary in SEOC places the long known origin of SEOC in doubt. Further there can be debates, regarding which MDCs or Stem Cells mutate to give rise to particular type of OC. Miles to go before an oncologist conquers OC (one of the deadliest cancers in world today), by improvements in diagnosis and treatment.

\section{Therapeutic implication of above discussions}

If my above hypothesis that Ovarian CSCs arise by the replacement of nuclei of oocyte by the nuclei of highly mutated MDCs (of ovary) is proved, this scientific discovery will open up a whole new world of research for the further possible discoveries of molecules/enzymes/proteins involved in the above mentioned natural process (that is counterpart of SCNT). Once known, the inhibitors of these molecules/enzymes/proteins could be designed as a robust vaccine against $\mathrm{OC}$, putting to end the need for all debate over the origin of $\mathrm{OC}$.

\section{Bibliography}

1. P K Sinha. "The common genesis of all cancers". Journal of Carcinogenesis and Mutagenesis 10 (2019): 342. 
2. P Dhawan., et al. "Cancer stem cells and colorectal cancer: An overview". Current Topics in Medicinal Chemistry 11 (2011): 1592-1598.

3. C A O'Brien., et al. "A human colon cancer cell capable of initiating tumor growth in immunodeficient mice". Nature 445.7123 (2007): 106-110.

4. E Zuber., et al. "Stem Cells in Ovarian Cancer and Potential Therapies". Proceedings of Stem Cell Research and Oncogenesis 8 (2020): e1001.

5. D Bhartiya and D Sharma. "Ovary does harbor stem cells - size of the cells matter!” Journal of Ovarian Research 13 (2020): 39.

6. SC Parte., et al. "Characterization of stem cell and cancer stem cell populations in ovary and ovarian tumors". Journal of Ovarian Research 11 (2018): 69.

7. I Virant-Klun and M Stimpfel. "Novel population of small tumorinitiating stem cells in the ovaries of women with borderline ovarian cancer". Scientific Reports 6 (2016): 34730.

8. M Stimpfel., et al. "Isolation, characterization and differentiation of cells expressing pluripotent/multipotent markers from adult human ovaries". Cell Tissue Research 354 (2013): 593607.

9. S Parte., et al. "Detection, characterization, and spontaneous differentiation in vitro of very small embryonic-like putative stem cells in adult mammalian ovary". Stem Cells Development 20.8 (2011): 1451-64.

10. Y L Clarkson., et al. "Initial characterisation of adult human ovarian cell populations isolated by DDX4 expression and aldehyde dehydrogenase activity". Scientific Reports 8 (2018): 6953.

11. I Virant-Klun., et al. "Isolation of small SSEA-4-positive putative stem cells from the ovarian surface epithelium of adult human ovaries by two different methods". BioMed Research International 2013 (2013): 690415.

12. M Wagner., et al. "Single-cell analysis of human ovarian cortex identifies distinct cell populations but no oogonial stem cells". Nature Communications 11 (2020): 1147.
13. X Fan., et al. "Single-cell reconstruction of follicular remodeling in the human adult ovary". Nature Communications 10 (2019): 3164.

14. K Nio., et al. "The evolving concept of liver cancer stem cells". Molecular Cancer 16 (2017): 4.

15. S Mukherjee., et al. "Plasticity in ovarian cancer: The molecular underpinnings and phenotypic heterogeneity". Journal of the Indian Institute of Science 100 (2020): 537-553.

16. J S Kim., et al. "Reprogrammed Pluripotent Stem Cells from Somatic Cells". International Journal of Stem Cells 4.1 (2011).

17. S Matoba and Y Zhang. "Somatic Cell Nuclear Transfer Reprogramming: Mechanisms and Applications". Cell Stem Cell 23.4 (2018): 471-485.

18. N K Suster., et al. "Presence and role of stem cells in ovarian cancer". World Journal of Stem Cells 11.7 (2018): 383-397.

\section{Volume 3 Issue 10 October 2021}

(C) All rights are reserved by Pushpam Kumar Sinha. 